\title{
Analysis Of Waveguide Whose Guiding Region Filled With Dielectric Material Bounded By Two Equiangular Spirals Separating It From Two Dielectric Cladding Regions
}

\author{
Alka Sharma \\ Department of Physics, J. N. P. G. College Lucknow University, Lucknow, India
}

\begin{abstract}
We Make an analytical study of a waveguide whose guiding cross-section is bounded by two equiangular spirals in which core and cladding regions are filled with different dielectric materials having refractive index $n_{1}$ and $n_{2}$ where $n_{1}>n_{2}$. The characteristic equation is derived under the scalar wave approximation for weak guidance. Dispersion curves and cutoff curve are also shown and interpreted. Unlike the planar waveguide, a nonzero cutoff is deduced which may be attributed to the curvature of the boundaries and the flare.
\end{abstract}

Keywords: Dispersion curves, weak guidance condition, waveguide with non-circular cross-section.

\section{Introduction}

In recent years optical waveguides with non-circular cross-section have received considerable attention. Among the non-circular cross-sections there are the triangular, elliptical, planar, polygonal and other more complicated shapes investigated [1-9]. Dielectric plane slab waveguides form the basic building blocks of a variety of devices in the field of integrated optics, laser beam technology, and microwave communications. Modal characteristics and attenuation properties of slab waveguide, multilayered waveguides and fibers have been studied extensively [10-12]. In the present paper we consider a waveguide whose guiding region is bounded by two equiangular spirals in which the core and the cladding regions are filled with different dieledric materials. If we consider a local region of this cross-section, we find that it resembles the cross-section of a distorted planar waveguide. The distortion has two aspects, namely, there is a curvature and there is also flare. This deformation may result from an external influence. For the analysis of such waveguide we use the scalar wave weak guidance approximation to obtain the characteristic equation. Dispersion curves for various modes are, however, obtained from the characteristic equation. The cutoff curve is also plotted after applying the cutoff condition. The analytical study of deformed planar waveguide with parabolic cylindrical boundaries [4] gave the result of bunching of modes. But in the proposed waveguide the deformation does not affect the discreteness of modes, it gives non- zero cutoff unlike the planar waveguide.

Theory

The cross-section of waveguide to be analysed is shown in figure (1). In the given figure three regions are shown and these are

Region (i): The first Cadding region

Region (ii): The Core region

Region (iii): The second Cladding region

The Polar equation of equiangular spirals is given by

$r=\xi e^{m \theta}$

Where $(r, \theta)$ are polar coordinates of a typical point on the spiral and $\xi$ and $m$ are two parameters.

Its normal curve is

$r=\eta e^{-\theta / m}$

To Introducing new coordinate system $(\xi, \eta, z)$ we obtain scale factors $h_{1}, h_{2}, h_{3}$. Thus

$h_{1}=\frac{1}{\sqrt{1+m^{2}}}\left(\frac{\eta}{\xi}\right)^{\frac{m^{2}}{1+m^{2}}}$

$h_{2}=\frac{m}{\sqrt{1+m^{2}}}\left(\frac{\xi}{\eta}\right)^{\frac{1}{1+m^{2}}}$

$h_{3}=1$

Using weak guidance approximation, the scalar wave equation of the wave function $\psi$ is given by

$\nabla^{2} \psi+\omega^{2} \mu_{0} \epsilon \psi=0$

In the new coordinate $\operatorname{system}(\xi, \eta, z)$, the scalar wave equation is 
$\left(\frac{1+m^{2}}{m}\right)\left(\frac{\xi}{\eta}\right)^{\frac{m^{2}}{1+m^{2}}}\left(\frac{\eta}{\xi}\right)^{\frac{1}{1+m^{2}}}\left[\frac{m}{\eta}\left(\left(\frac{\partial \varphi}{\partial \xi}+\xi \frac{\partial^{2} \varphi}{\partial \xi^{2}}\right)+\frac{1}{m \xi}\left(\frac{\partial \varphi}{\partial \eta}+\eta \frac{\partial^{2} \varphi}{\partial \eta^{2}}\right)\right)\right]+\frac{\partial^{2} \varphi}{d z^{2}}+\omega^{2} \mu_{0} \epsilon \psi=0$

To solve above equation the separation of variables technique can be applied

$$
\psi(\xi, \eta, z)=F_{1}(\xi) \cdot F_{2}(\eta) e^{i \beta z}
$$

Where $\beta$ is the $\mathrm{z}$-component of propagation vector. Using expression (5) we get rather complicated equation, for simplification consider an approximation namely $\mathrm{m}<<<1$, we obtain following two equations.

$\xi^{2}\left(1+m^{2}\right) \frac{d^{2} F_{1}}{d \xi^{2}}+\left(1+m^{2}\right) \xi \frac{d F_{1}}{d \xi}+\left[\left(\omega^{2} \mu_{0} \epsilon-\beta^{2}\right) \xi^{2}-k^{2}\right] F_{1}=0$

and

$\eta^{2} \frac{d^{2} F_{2}}{d \eta^{2}}+\eta \frac{d F_{2}}{d \eta}+\frac{m^{2}}{1+m^{2}} k^{2} F_{2}=0$

Where $\mathrm{k}$ is the unknown constant. Since $\mu$ and $\epsilon$ are absent in equation (7) it is not physically relevance. Equation (6) can be written in the given form

$\frac{d^{2} F_{1}}{d \xi^{2}}+\frac{1}{\xi} \frac{d F_{1}}{d \xi}+\left[u^{\prime 2}-\frac{n^{2}}{\xi^{2}}\right] F_{1}=0$

Where

$\frac{\omega^{2} \mu_{0} \epsilon_{1}-\beta^{2}}{1+m^{2}}=u^{\prime 2} \quad$ and $\quad \frac{k^{2}}{1+m^{2}}=n^{2}$

The equation (8) is an ordianary Bessel differential equation. The solutions of this equation are $J_{n}\left(u^{\prime} \xi\right)$ and $Y_{n}\left(u^{\prime} \xi\right)$. Where $Y_{n}\left(u^{\prime} \xi\right)$ is the Bessel function of the second kind, $\mathrm{n}$ can take even non-integral value.

The wave equation for the cladding region

$\frac{d^{2} G_{1}}{d \xi^{2}}+\frac{1}{\xi} \frac{d G_{1}}{d \xi}-\left[w^{2}+\frac{n^{2}}{\xi^{2}}\right] G_{1}=0$

Here $w^{\prime 2}=\frac{\beta^{2}-\omega^{2} \mu_{0} \epsilon_{2}}{1+m^{2}}$

This equation represents the modified Bassel differential equation. The two independent solutions of equation (9) will be $I_{n}\left(w^{\prime} \xi\right)$ and $K_{n}\left(w^{\prime} \xi\right)$.

Thus the acceptable solutions in the core and cladding regions are:-

(i) In region I ie (cladding region) the acceptable solution is $I_{n}\left(w^{\prime} \xi\right)$, due to increasing nature.

(ii) In region II (core region) the acceptable solutions are $J_{n}\left(u^{\prime} \xi\right)$ and $Y_{n}\left(u^{\prime} \xi\right)$, due to oscillating nature.

(iii) In the third region the acceptable solution is $K_{n}\left(w^{\prime} \xi\right)$, due to its decreasing nature.

To obtain the characteristic equation for the above mentioned waveguide, apply boundary conditions at two boundaries ie at $\xi_{1}=a$ and $\xi_{2}=b$

These conditions are

$$
\begin{aligned}
& \begin{array}{c}
A J_{n}\left(u^{\prime} \xi\right)+B Y_{n}\left(u^{\prime} \xi\right)=C I_{n}\left(w^{\prime} \xi\right) \\
J_{n}^{\prime}\left(u^{\prime} \xi\right)+B u^{\prime} Y_{n}^{\prime}\left(u^{\prime} \xi\right)=c w^{\prime} I_{n}^{\prime}\left(w^{\prime} \xi\right)
\end{array} \mid \quad \xi=a \\
& \begin{array}{l}
A J_{n}\left(u^{\prime} \xi\right)+B Y_{n}\left(u^{\prime} \xi\right)=F K_{n}\left(w^{\prime} \xi\right) \\
J^{\prime}\left(u^{\prime} \xi\right)+B u^{\prime} Y^{\prime}{ }_{n}\left(u^{\prime} \xi\right)=F w^{\prime} K^{\prime}{ }_{n}\left(w^{\prime} \xi\right)
\end{array} \mid \quad \xi=b
\end{aligned}
$$

Where $n=\frac{k}{\sqrt{1+m^{2}}}$

Since for convenience, we consider the value of $\mathrm{k}$ as 1 and keep a fixed value of $\mathrm{m}=1.12$. For these, $n=\frac{2}{3}$. Using boundary conditions $\left[\right.$ For $\left.n=\frac{2}{3}, u^{\prime}=\frac{2}{3} u, w^{\prime}=\frac{2}{3} w\right]$ following equations are obtained.

$$
\begin{aligned}
& \begin{array}{c}
A J_{2 / 3}\left(\frac{2}{3} u \xi\right)+B Y_{2 / 3}\left(\frac{2}{3} u \xi\right)=C I_{2 / 3}\left(\frac{2}{3} w \xi\right) \\
J_{2 / 3}^{\prime}\left(\frac{2}{3} u \xi\right)+B \frac{2}{3} u Y_{2 / 3}^{\prime}\left(\frac{2}{3} u \xi\right)=C_{\frac{3}{3}}^{2} w I_{2 / 3}^{\prime}\left(\frac{2}{3} w \xi\right)
\end{array} \mid \xi=a \\
& A J_{2 / 3}\left(\frac{2}{3} u \xi\right)+B Y_{2 / 3}\left(\frac{2}{3} u \xi\right)=F K_{2 / 3}\left(\frac{2}{3} w \xi\right) \\
& A \frac{2}{3} u J_{2 / 3}^{\prime}\left(\frac{2}{3} u \xi\right)+B \frac{2}{3} u Y_{2 / 3}^{\prime}\left(\frac{2}{3} u \xi\right)=F_{\frac{3}{3}}^{2} w K_{2 / 3}^{\prime}\left(\frac{2}{3} w \xi\right) \mid \xi=b
\end{aligned}
$$

The set of these four equations with unknowns A, B, C, F has a non-trivial solution if the determinant formed by the coefficients of these unknown constant is zero.

$$
\Delta=\left|\begin{array}{llrr}
J_{2 / 3}\left(\frac{2}{3} u a\right) & Y_{2 / 3}\left(\frac{2}{3} u a\right) & -I_{2 / 3}\left(\frac{2}{3} w a\right) & 0 \\
\frac{2}{3} u J_{2 / 3}^{\prime}\left(\frac{2}{3} u a\right) & \frac{2}{3} u Y_{2 / 3}^{\prime}\left(\frac{2}{3} u a\right) & -\frac{2}{3} w I_{2 / 3}^{\prime}\left(\frac{2}{3} w a\right) & 0 \\
J_{2 / 3}\left(\frac{2}{3} u b\right) & Y_{2 / 3}\left(\frac{2}{3} u b\right) & 0 & -K_{2 / 3}\left(\frac{2}{3} w b\right) \\
\frac{2}{3} u J_{2 / 3}^{\prime}(u b) & \frac{2}{3} u Y_{2 / 3}^{\prime}\left(\frac{2}{3} u b\right) & 0 & -\frac{2}{3} w K_{2 / 3}^{\prime}\left(\frac{2}{3} w b\right)
\end{array}\right|=0
$$


On expanding this determinant we can obtain the characteristic equation

$$
\Delta=0
$$

Cutoff condition can be achieved from the characteristic equation taking $\mathrm{w} \rightarrow 0$ in the cladding region.

$$
\Delta_{1}=\left|\begin{array}{lllr}
J_{2 / 3}\left(\frac{2}{3} u a\right) & Y_{2 / 3}\left(\frac{2}{3} u a\right) & -1 & 0 \\
\frac{2}{3} u J_{2 / 3}^{\prime}\left(\frac{2}{3} u a\right) & \frac{2}{3} u Y_{2 / 3}^{\prime}\left(\frac{2}{3} u a\right) & -\frac{4}{3} & 0 \\
J_{2 / 3}\left(\frac{2}{3} u b\right) & Y_{2 / 3}\left(\frac{2}{3} u b\right) & 0 & -1 \\
\frac{2}{3} u J_{2 / 3}^{\prime}\left(\frac{2}{3} u b\right) & \frac{2}{3} u Y_{2 / 3}^{\prime}\left(\frac{2}{3} u b\right) & 0 & -\frac{4}{3}
\end{array}\right|=0
$$

Cut of Equation is

$$
\Delta_{1}=0
$$

The solution of this equation will yield the cut-off $\beta$-values corresponding to the guided modes.

\section{Numerical Computation}

We are now in a position to make some numerical estimates of the modal properties of a fiber with a guiding region bounded by two equiangular spirals. For this the parameters have been given the following values. We take $n_{1}=1.50$ (core refractive index) $n_{2}=1.48$ (cladding refractive index) and operating wavelength $\lambda_{0}=1.55 \mu$. All of the significant modal properties are obtainable from characteristic equation (10).

In order to obtain the dispersion curves from characteristic equation (10) we plot a graph between allowed $\beta$ values and left hand side of the characteristic equation (10) at fixed values of $a$ and $b$. It is known that $\beta$ values lie in the range $n_{1} k_{0}>\beta>n_{2} k_{0}$, where $k_{0}=\frac{2 \pi}{\lambda_{0}}$. For convenience, we again consider the value of a as $2 \mu$, whereas the size parameter $\mathrm{b}$ is allowed to change from $3 \mu$ to $20 \mu$. Thus we plot a graph between the allowed $\beta$ values and left hand side of characteristic equation (10) for different values of (b-a) in which ' $a$ ' is fixed and $b$ is allowed to change. From these graphs we can obtain the cutoff $\beta$ values corresponding to the intersections of the curves with the $\beta$ axis for different modes. These cutoff $\beta$ values are plotted against the ' $V$ ' parameter for each mode. The ' $\mathrm{V}$ ' parameter defined as $V=\frac{2 \pi}{\lambda_{0}}(b-a) \sqrt{n_{1}{ }^{2}-n_{2}{ }^{2}}$. It is reasonable to plot a graph between the ' $b$ ' parameter (normalised propagation constant) versus $\mathrm{V}$ parameter rather than a graph between cutoff $\beta$ values and V parameter, where $b_{n o r}^{\prime}=\frac{\left(\frac{\beta}{k_{0}}\right)^{2}-n_{1}{ }^{2}}{n_{1}{ }^{2}-n_{2}{ }^{2}}$. These dispersion curves are shown in fig (2). To obtain modal cutoff curve for this waveguide we plot a graph between $\Delta_{1}$ and $\mathrm{V}$ parameter $(V=u(b-a))$ represented by fig (3). All these calculations are done for a fixed value of $m=1.12$ and for a fixed value of $k=1$ (lowest mode), so $\mathrm{n}$ is equal to $2 / 3$.

\section{Result And Discussion}

From the above computational analysis we are in a position to make several interesting results about the presented optical waveguide whose guiding region is bounded by two equiangular spirals with dielectric core-dielectric cladding. The dispersion curves (shown in figure 2) give us information about the number of modes which can propagate through the considered waveguide. When $\mathrm{V}<2$, the waveguide sustains only a single mode. The number of modes is increased with the value of $\mathrm{V}$ parameter as expected. When $\mathrm{V}<7$ this waveguide allows to propagate two modes. When $\mathrm{V}<12$ and $\mathrm{V}<16$ the number of possible modes sustained by the waveguide is three and four respectively. The results obtained from dispersion curves are consistent with those obtained from the cutoff curve which is shown in fig (3). Since the cutoff curve is obtained by the approximation $w \rightarrow 0$, in the characteristic equation, there is some minor difference in the cutoff $\mathrm{V}$ values which are obtained from the cutoff curve and those found from dispersion curves. The dispersion curves, however are more realiable than cutoff curve. The considered waveguide can be looked upon as distorted planar waveguide with a curvature and flare. If we compare the dispersion curves for planar waveguide with considered waveguide, the modes of such a distorted planar waveguide are discrete like planar waveguide and tend towards higher $\mathrm{V}$ values. It is well known that for a planar waveguide the cutoff values for successive TM and TE modes are obtained at $\mathrm{V}_{\mathrm{C}}=\mathrm{m} \pi$, where $\mathrm{m}=0,1,2, \ldots$ etc. In the considered waveguide we find form dispersion curves that the lowest cutoff value is near $\mathrm{V} \approx 1$ which is different from zero. This dissimilarity with planar waveguide may be attributed to the curvature and flare of the boundaries. Another interesting thing is that when planar waveguide deformed with parabolic cylindrical boundaries [4] the dispersion curves show the bunching of modes, unlike the planar waveguide, but the proposed deformation of planar waveguide does not change the discreteness of modes, it only yield a non-zero cutoff which is not seen in case of planar waveguide. 


\section{Acknowledgment}

It is a great pleasure to contribute this article in honour of the late Prof. P. Khastgir, who has been a kind, generous and inspiring teacher.

\section{References}

[1] R.B. Dyott, "Cutoff of the first higher order modes in elliptical dielectric waveguide: an experimental approach", Electron. Lett. 26, 1990, pp. $1721-1723$

[2] V. Misra, P.K. Choudhury, P. Khastgir, S.P. Ojha, "Modal propagation analysis of a waveguide with a regular pentagonal crosssection with conducting and non-conducting boundaries.”, Microwave Opt. Technol. Lett 8, 1995, pp. $280-282$.

[3] A. Sharma, "Modal dispersion curves of an optical waveguide with a core cross-section partially bounded by one turn of an equiangular spiral under weak guidance approximation, Optik" 122, 2011, pp.1535-1537.

[4] P.K. Choudhary, P. Khastgir, S.P. Ojha, L.K. Singh, "Analysis of the guidance of electromagnetic wave by a deformed planar waveguide with parabolic cylindrical boundaries", J. Appl Phys. 71, 1992, pp. 5685-5688.

[5] V. Singh, B. Prasad, S.P. Ojha, "Weak guidance modal analysis and dispersion curves of an infrared lightguide having a core crosssection with new type of asymmetric loop boundary", Opt. Fiber Technol. (USA) 6, 2000, pp. 290-298.

[6] V. Singh, B. Prasad, S.P. Ojha, "A comparative study of modal characteristic and waveguide dispersion of optical waveguide with three different closed loop cross-sectional boundaries", Optik 115, 2004, pp. 281-284.

[7] H.P. Singh, V. Singh, V.P. Arora, "Modal analysis and dispersion curves of a curvilinear triangular cored lightguide using Goell's Point matching method", Optik 119, 2008, pp. 29-33.

[8] A.K. Mishra, P.C. Pandey, D. Kumar, O.N. Singh, "An analytical study of modal dispersion characteristics of helically clad crystalcored optical fiber", Optik 124(17), 2013, pp. 2611-2617.

[9] A.K. Shahi, V. Singh, S.P. Ojha, "Towards Dispersion Characteristics for a new unconventional metal-clad optical waveguide", Microwave and Optical Technology Letters Vol. 49(11), 2007, pp. 2709-2713.

[10] H.G. Unger, "Planar Optical Waveguide and Fibers", Clarendon, Oxford, 1977, Chap. 2.

[11] W.C. Borland, D.E. Zelmon, C.J. Radens, J.T. Boyd and H.E. Jackson, IEEE J. Quantum Electron, QE-23 ,1987, pp. 1172.

[12] V.K. Chaubey, K.K. Dey, S.P. Ojha and P. Khastgir, Can. J. Phys. 66, 1988, pp. 796

\section{Figure Caption}

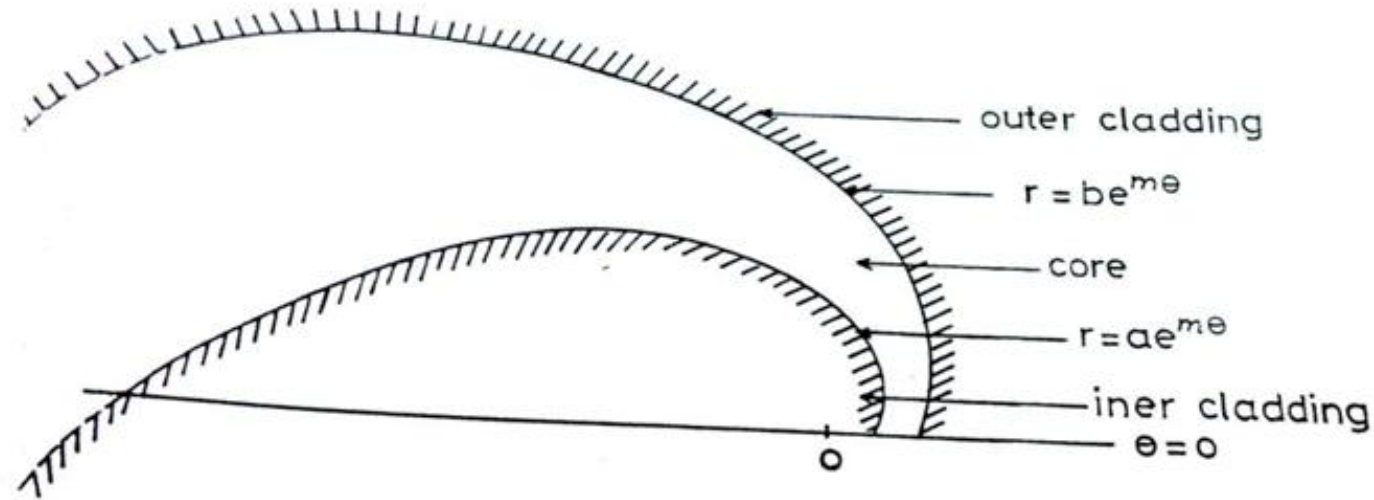

Fig 1: The guiding region cross-section bounded by two equiangular spirals.

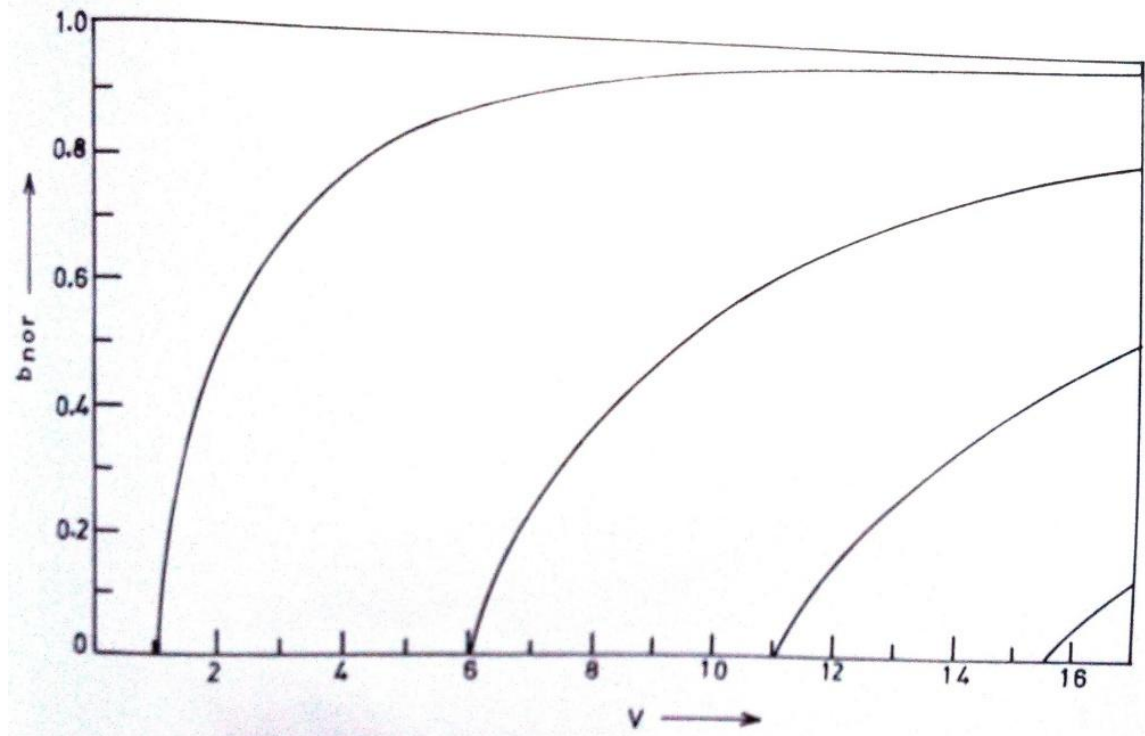

Fig 2: dispersion curves of some lower order modes for $a=2 \mu$ (the parameter $b$ is allowed to change). 


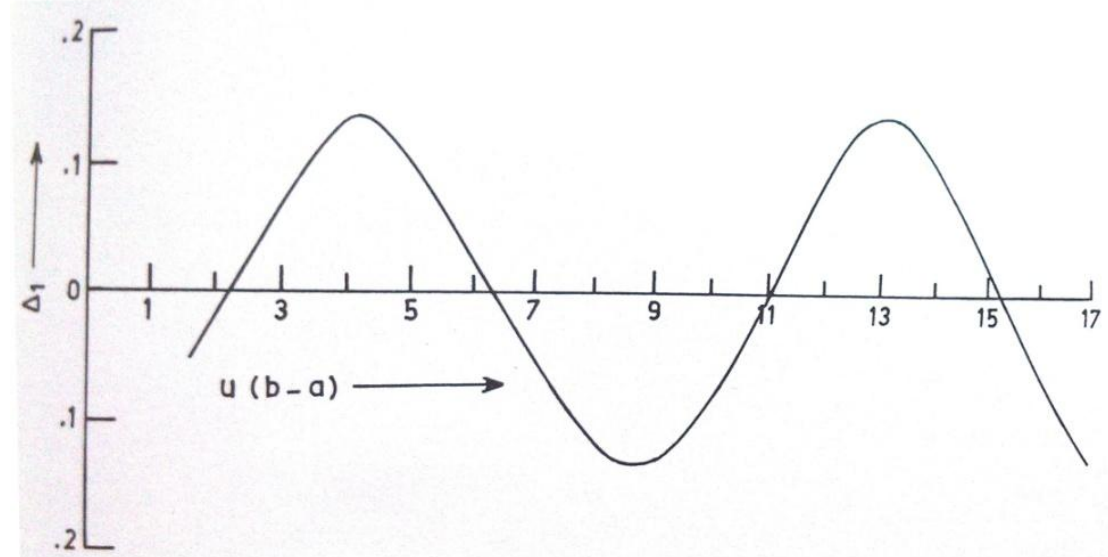

Fig 3: The plot of left hand side $\Delta_{1}$ of equation (11) against u (b-a). 